\title{
REVIEW
}

\section{Heparin-induced thrombocytopenia in the ICU: an overview}

\author{
Yasser Sakr* \\ This article is one of eleven reviews selected from the Annual Update in Intensive Care and Emergency Medicine 2011 (Springer Verlag) and \\ co-published as a series in Critical Care. Other articles in the series can be found online at http://ccforum.com/series/annual. Further \\ information about the Annual Update in Intensive Care and Emergency Medicine is available from http://www.springer.com/series/8901
}

\begin{abstract}
Introduction
Heparin, a negatively charged glycosaminoglycan $(3,000-$ $30,000 \mathrm{Da}$ ), is an anticoagulant released by mast cells and basophils during the normal clotting process [1]. Heparin is widely used for the treatment and prophylaxis of thromboembolic diseases in medical and surgical patients [1]. Heparin-induced thrombocytopenia (HIT) is one of the most serious adverse events associated with this drug. HIT is an immune-mediated, prothrombotic complication that occurs with unfractionated heparin (UFH) and to a lesser extent with low-molecular-weight heparin (LMWH) [2]. The fundamental paradox of HIT results from a platelet-activating immune response triggered by the interaction of heparin with a specific platelet protein, platelet factor 4 (PF4) [3].

In this chapter, we review current knowledge about the pathophysiology, epidemiology, clinical manifestations, and treatment of HIT in the intensive care unit (ICU).
\end{abstract}

\section{Pathophysiology of HIT}

Heparin causes mild platelet aggregation in vivo, especially in patients with activated platelets, resulting in increased platelet sequestration in the spleen and thrombocytopenia [1]. Thrombocytopenia can be triggered via non-immune and immune mechanisms. Clinically, two types of HIT can be differentiated: HIT type I, a benign non-immune condition; and HIT type II, an immunemediated syndrome caused by an antibody to the PF4/ heparin complex.

Non-immune HIT, or HIT type I, is a self-limiting condition without any major complications that occurs in $10-30 \%$ of patients within 4 days after exposure to heparin. Heparin binds to PF4 with high affinity and

*Correspondence: Yasser-Sakr@med.uni-jena.de Department of Anesthesiology and Intensive Care, Friedrich-Schiller-University, Erlanger Allee 103, 07743 Jena, Germany

(C) 2011 Springer-Verlag Berlin Heidelberg.

This work is subject to copyright. All rights are reserved, whether the whole or part of the material is concerned, specifically the rights of translation, reprinting, reuse of illustrations, recitation, broadcasting, reproduction on microfilm or in any other way, and storage in data banks. Duplication of this publication or parts thereof is permitted only under the provisions of the German Copyright Law of September 9 , 1965 , in its current version, and permission for use must always be obtained from Springer-Verlag. Violations are liable for prosecution under the German Copyright Law. inhibits adenylcyclase. This leads to a decrease in intracellular cyclic adenosine monophosphate (cAMP) levels with subsequent reduction in the platelet activation threshold and mild platelet aggregation and thrombocytopenia [4,5]. HIT type I may occur in patients with sepsis, burn injuries, and vascular diseases, probably due to platelet hyperreactivity in these conditions $[4,5]$. Thrombocytopenia in HIT type I is usually mild and

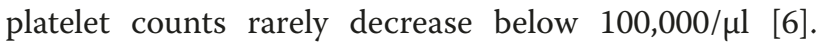
Heparin administration should be continued and no specific therapy is required.

Immune-mediated HIT type II is a disorder initiated by an immunological response to heparin exposure and is characterized by an absolute or relative thrombocytopenia with a paradoxically increased incidence of thrombosis (Fig. 1) [1]. The major antigen responsible for this syndrome is PF4, which is synthesized by megakaryocytes and stored in platelet $\alpha$-granules. Upon platelet activation, PF4 is released and binds anionic glycosaminoglycans on cell surfaces. The main function of PF4 is to inhibit the formation of megakaryocytes and angiogenesis, as well as modulating the immune response. Considerable amounts of PF4 are released after trauma, inflammation, surgical trauma, and in neoplasm [7]. In HIT type II, heparin infusion displaces PF4 and produces structural changes on it, leading to the formation of a PF4/heparin complex. This complex is recognized as a 'foreign' antigen and triggers an immune response, which is characterized by the release of IgG antibodies that bind to the PF4/heparin complexes with subsequent clustering of the platelet Fc-receptors (FcXRIIa, FcXRIIIa) resulting in platelet activation. This may lead to overt arterial thrombosis, historically, called "the white clot syndrome". Activated platelets can also fragment into prothrombotic microparticles and stimulate venous thrombosis $[5,8]$. In addition, HIT antibodies may bind to Fc receptors on monocytes which produces \\ C Biomed Central}




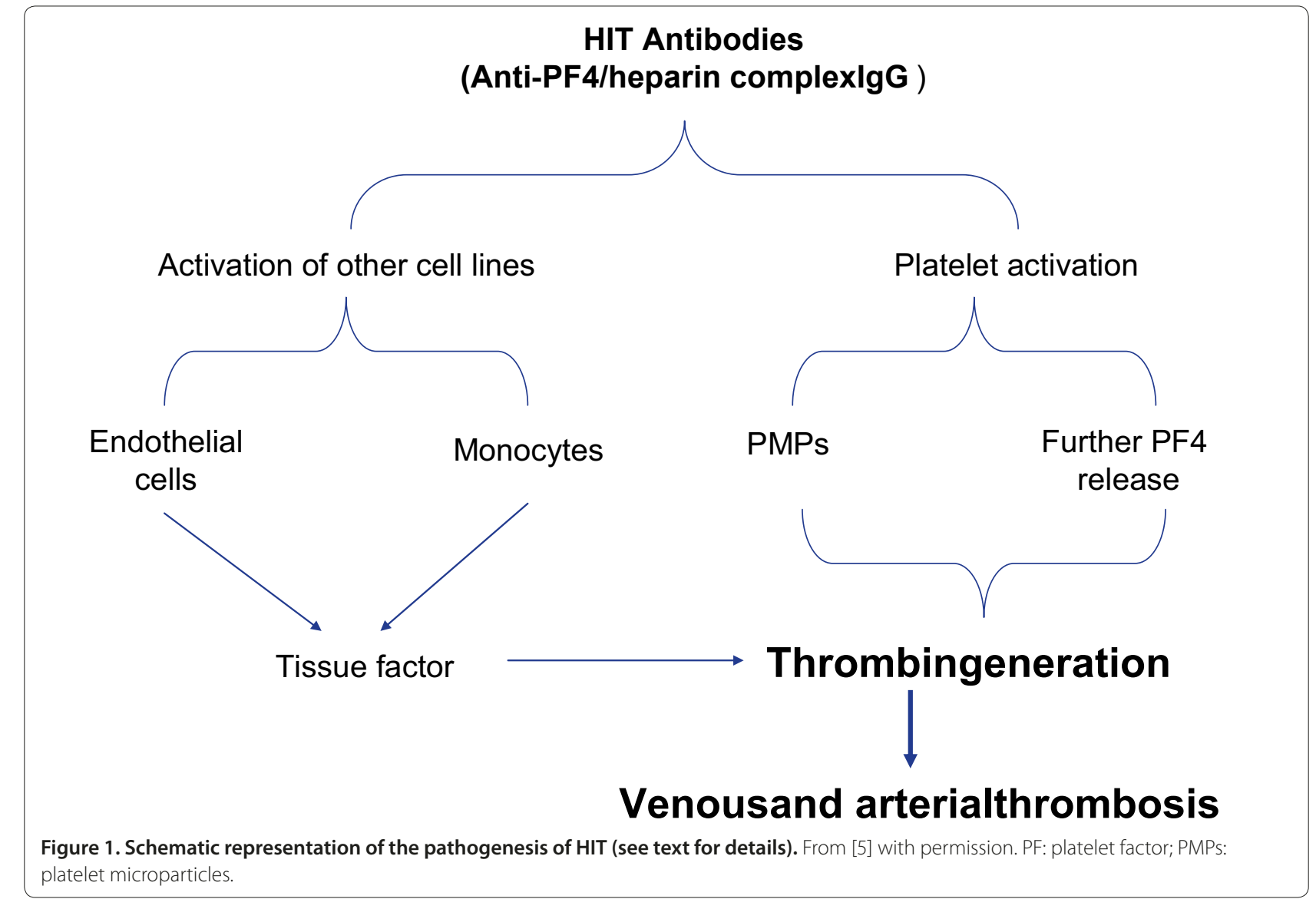

significant quantities of tissue factor, stimulating thrombosis [5,9]. HIT antibodies may promote thrombosis through platelet adhesion to the vessel wall and formation of platelet-leukocyte aggregates [5,10]. Davidson et al. [11] reported elevated levels of von Willebrand factor and soluble thrombomodulin in patients with HIT type II, suggesting that endothelial cell damage with the consecutive loss of its physiologic antithrombotic properties may contribute to the thrombotic risk.

Heparin molecules bind PF4 in proportion to the length of the polysaccharide chain. This explains the higher frequency of HIT among patients treated with UFH than among those treated with LMWH [12]. The amount of anti-PF4/heparin antibodies produced is determined not only by the dose and structure of heparin but also by the amount of circulating PF4. In some clinical situations, such as cardiac surgery, the relatively abundant circulating PF4 and heparin increase the risk of immunization [7]. PF4 bound in vivo to cell surface glycosaminoglycans can be immunogenic and could explain why healthy individuals may be positive for anti-PF4/heparin antibodies [13]. In fact, not all patients who have heparin antibodies develop platelet activation and clinically relevant HIT. After termination of heparin therapy, the platelet count increases within 4 to 14 days [14]. HIT antibodies are transient, generally disappearing within 4 months. [15]

HIT type II is the most important clinical entity and will be discussed in the following sections. For simplicity, we will refer to HIT type II simply as HIT.

\section{Epidemiology}

The frequency of HIT in heparin-exposed patients is highly variable. Heparin preparation is one influential factor with bovine UFH being the most common trigger followed by porcine UFH [12]. HIT occurs less commonly in patients receiving LMWH. The incidence of HIT is $1-5 \%$ when UFH is used but $<1 \%$ with LMWH [12]. Females are more likely to develop HIT than males and postoperative patients have a higher incidence of HIT than have medical ICU patients [16]. Heparin dosage also plays an important role. Prophylactic doses of heparin increase the risk of antibody formation, whereas clinical manifestations occur more in patients receiving therapeutic doses [16]. Only a small proportion, at most 5\% to $30 \%$, of patients who form HIT-IgG will develop clinical HIT $[12,17]$.

The incidence of HIT varies from $0 \%$ in pregnant women receiving $\mathrm{LMWH}$ to $5 \%$ in patients undergoing 
orthopedic surgery receiving UFH [18]. Despite the relatively high prevalence of anti-PF4/heparin antibodies in patients undergoing cardiac surgery, the incidence of HIT in this patient population is about $2.4 \%$ [18]. The formation of anti-PF4/heparin antibodies varies from 2 to $5 \%$ in cardiology patients, from 15 to $30 \%$ in patients undergoing orthopedic surgery, and up to 30 to $70 \%$ in patients undergoing cardiac surgery [19]. Several studies have assessed the frequency of HIT in ICU patients [3]; the incidence of HIT in ICU patients is generally less than $2 \%$.

\section{Clinical manifestations}

HIT is a clinicopathological syndrome with one or more clinical events (thrombocytopenia with or without thrombosis) temporally related to heparin administration and caused by HIT antibodies [20]. The clinical manifestations of HIT are discussed below.

\section{Onset}

In patients with HIT, thrombocytopenia typically occurs 5-10 days after initiation of heparin therapy (typical onset HIT) as the immune system requires several days to produce sufficient amounts of anti-PF4/heparin antibodies [21]. Thrombocytopenia that occurs more than 10 days after exposure to heparin is probably caused by other factors, such as sepsis. In some exceptional cases, invasive procedures, such as surgical interventions, may promote seroconversion and release of PF4 after long periods of exposure to heparin [21].

In the so called 'rapid onset' HIT, thrombocytopenia occurs within 24 hours of exposure to heparin, mostly due to the presence of anti-PF4/heparin antibodies after prior exposure to heparin within the previous 100 days [15]. The onset of HIT in these cases is usually accompanied by fever, shivering, and skin lesions at the injection sites within 30 minutes after heparin administration [3]. Some patients also develop acute respiratory or cardiac dysfunction, manifested as hypertension, tachycardia, angina pectoris, or dyspnea. These manifestations may suggest pulmonary embolism because of the sudden pronounced platelet activation $[3,15]$.

In some patients, HIT may occur after termination of heparin therapy. Thrombotic events or low platelet counts may draw attention to the presence of HIT. This 'delayed onset' HIT is associated with large numbers of anti-PF4/heparin antibodies, which lead to platelet activation in the absence of heparin [3]. This entity may be clinically relevant in patients who are discharged early from the hospital after surgical interventions.

\section{Thrombocytopenia}

Thrombocytopenia is the first sign of HIT in $85-90 \%$ of patients who have a decrease in platelet count below $150,000 / \mu \mathrm{l}$ or a reduction of more than $50 \%$ from the baseline platelet count [3]. Platelet count usually falls to values between 40,000 to $80,000 / \mu \mathrm{l}$. In only $5-10 \%$ of cases, does the platelet count reach a nadir below 20,000/ $\mu \mathrm{l}$ [22], and in such cases other possible causes of thrombocytopenia should be considered.

Thrombocytopenia is a common laboratory abnormality in critically ill patients. Prospective data from 329 adult surgical ICU patients during one year showed that $41.3 \%$ had a platelet count less than $150,000 / \mu$ l at some point [23]. The most common etiology of thrombocytopenia in critical illness is sepsis (around 48\%), although $25 \%$ of ICU patients have more than one cause [24]. Drug-induced thrombocytopenia must be considered, since several medications can cause thrombocytopenia and critically ill patients usually receive numerous medications [25]. Possible causes of thrombocytopenia are shown in Table 1.

\section{Thrombotic complications}

HIT is associated with thrombotic complications in $30-70 \%$ of cases. These may develop without significant thrombocytopenia in $15 \%$ of patients [18]. In patients with symptomatic deep vein thrombosis (DVT) after initiation of heparin therapy, HIT cannot be excluded even in the absence of thrombocytopenia. Thrombotic events occur around three days before the onset of thrombocytopenia in $40 \%$ of HIT patients [26]. The risk of thrombosis correlates, however, to the magnitude of relative thrombocytopenia [26].

The most common thrombotic complications in patients with HIT include DVT (50\%) and pulmonary embolism (25\%) [27]. Other less common complications include myocardial infarction, cardiovascular accidents, arterial occlusive lower limb ischemia, sinus vein thrombosis, mesenteric venous or arterial occlusion, and skin necrosis [28]. Venous thrombosis is 4 to 10 times higher than arterial thrombosis [3,27].

\section{Other complications of HIT}

The risk of bleeding in patients with HIT is relatively low, even at a platelet count of less than 20,000/ $\mu$ l [3]. However, bleeding can occur due to thrombocytic dysfunction, such as in patients with uremia. Wester et al. [29] compared 20 patients with HIT to 20 ICU patients without HIT as a control group. Although patients with HIT had a higher incidence of bleeding than the control group ( 85 vs. $35 \%$ ), bleeding in the HIT patients occurred under heparin therapy and was not directly related to thrombocytopenia.

In a median of 8 days after the onset of heparin therapy, 10 to $20 \%$ of patients with HIT develop skin lesions in the form of erythematous nodules, subcutaneous plaques, or necrotic lesions [30]. Skin lesions occur equally after treatment with UFH or LMWH [30]. 
Table 1. Differential diagnosis of thrombocytopenia in the ICU

Sepsis and healthcare-associated infections

Drug-induced thrombocytopenia: e.g., GP IIb/IIla inhibitor, thrombolytic agents, and antibiotics (e.g., vancomycin)

Perioperative fluid resuscitation

Disseminated intravascular coagulation (DIC)

Massive transfusion

Intravascular devices: ECMO, IABP, LVAD and pulmonary catheter

Liver disease/hypersplenism

Pulmonary embolism

Immune thrombocytopenias

Diabetic ketoacidosis

Cancer-associated DIC, primary bone marrow disorder

Antiphospholipid syndrome and systemic lupus erythematosis

EDTA-induced pseudothrombocytopenia

ECMO: extracorporeal membrane oxygenation; IABP: intra-aortic balloon pump; LVAD: left ventricular assist device; EDTA: ethylenediaminetetraacetic acid

\section{Scoring system for HIT}

The ' 4 T's' scoring system is based on thrombocytopenia, timing of onset, thrombosis, and absence of other causes (Table 2) and allows evaluation of the pretest probability of HIT [31]. Patients with low pretest scores (< 4 points) are unlikely to be positive for HIT antibodies (0 to 1.6\%), whereas patients with intermediate (4-5 points) and high (> 5 points) scores are more likely to test positive (21.4\% to $100 \%$ ) [32]. The evaluation of this scoring system showed a high negative predictive value in the general population and in ICU patients, with low scores being suitable for ruling out HIT in most clinical situations [32].

\section{Laboratory diagnosis of HIT}

In patients with suspected HIT, diagnosis can be established by laboratory testing for the presence of HIT antibodies. Two types of assays are available: Functional and antigen assays.

\section{Functional assays}

These assays are based on in vitro activation of platelets as evidence for the presence of relevant IgG-HIT antibodies [20]. Heparin-induced platelet activation (HIPA) and serotonin release assays (SRA) are examples of these tests. Washed platelets from healthy volunteers are mixed with patient serum and then incubated with low and high concentrations of heparin. In the presence of HIT antibodies, platelets are activated in low concentrations of heparin. This activation can be detected using radioactive serotonin (e.g., SRA) or visually (e.g., HIPA) [19].

The strength of functional assays is their very high specificity, if the appropriate controls are performed. Whether these tests can reach $100 \%$ sensitivity depends on the 'gold standard' against which they are compared. Moreover, functional assays are technically demanding (selected platelet donors, washed platelets, internal controls, radioactivity), have a high turn-around time, and are performed by only a minority of experienced laboratories [19].

\section{Antigen assays}

Enzyme-linked immunoassays (ELISA) are the tests most commonly used to detect HIT antibodies. These assays non-specifically detect IgG, IgA, and IgM antibodies against PF4/heparin. The results are analyzed photometrically and an optical density higher than 0.4 is considered as positive [3]. ELISA is highly sensitive due to its ability to detect a broad range of HIT antibodies. However, the specificity of ELISA is lower compared to functional assays [3].

Particle gel immunoassay (PaGIA) is another antigen assay that may be used to detect HIT antibodies. The IDheparin/PF4 antibody test (ID-Micro Typing System DiaMed $^{\circ}$ ) is a PaGIA, in which a PF4/heparin-coated synthetic polymer is used. The agglutination of HIT antibodies in patient serum leads to the formation of bands on the gel matrix. The results can be obtained after 20 minutes. The sensitivity and specificity of this test lies between the functional tests and the ELISA [33]. PaGIA may be used, therefore, as a rapid screening test, pending the results of functional assays [33].

The particle immunofiltration assay (PIFA) is another screening test which involves the use of PF4-coated colored polymer particles. This test, however, has relatively low performance in terms of specificity and sensitivity [34].

\section{Interpretation of the results of laboratory assays}

As mentioned above, antigen assays are highly sensitive. These tests can be used, therefore, to exclude the 
Table 2. The 4 T's score [32]

\begin{tabular}{|c|c|c|c|}
\hline \multirow[b]{2}{*}{4 T Category } & \multicolumn{2}{|r|}{ Score } & \multirow[b]{2}{*}{0} \\
\hline & 2 & 1 & \\
\hline Thrombocytopenia & $\begin{array}{l}\text { Platelet count decrease }>50 \% \text { or } \\
\text { platelet nadir } \geq 20 \times 10^{9} / \mathrm{L}\end{array}$ & $\begin{array}{l}\text { Platelet count decrease } 30-50 \% \text { or } \\
\text { platelet nadir } 10-19 \times 10^{9} / \mathrm{L}\end{array}$ & $\begin{array}{l}\text { Platelet count decrease }<30 \% \text { or } \\
\text { platelet nadir }<10 \times 10^{9} / \mathrm{L}\end{array}$ \\
\hline $\begin{array}{l}\text { Time to platelet count } \\
\text { decrease* }^{*}\end{array}$ & $\begin{array}{l}\text { Clear onset between days } 5-10 \text { or } \\
\text { platelet count decrease } \leq 1 \text { day (prior } \\
\text { heparin exposure within } 100 \text { days) }\end{array}$ & $\begin{array}{l}\text { Consistent with decrease between } \\
\text { days } 5-10 \text {, but not clear (e.g., missing } \\
\text { platelet counts); onset after day } 10\end{array}$ & $\begin{array}{l}\text { Platelet count decrease }<4 \text { days } \\
\text { without recent exposure }\end{array}$ \\
\hline $\begin{array}{l}\text { Thrombosis or other } \\
\text { sequelae }\end{array}$ & $\begin{array}{l}\text { New thrombosis (confirmed); skin } \\
\text { necrosis; acute systemic reaction } \\
\text { postintravenous heparin }\end{array}$ & $\begin{array}{l}\text { Progressive or recurrent thrombosis; } \\
\text { non-necrotizing (erythematous) skin } \\
\text { lesions; suspected thrombosis (not proven) }\end{array}$ & None \\
\hline $\begin{array}{l}\text { Other causes of } \\
\text { thrombocytopenia }\end{array}$ & None apparent & Possible & Definite \\
\hline
\end{tabular}

*First day immunizing heparin exposure considered day zero; the day the platelet count begins to decrease is considered the day of onset of thrombocytopenia.

presence of HIT. However, positive results do not confirm the diagnosis or reflect the risk of thrombotic events [35]. Increasing the ELISA optical density threshold from 0.4 to 1 may increase the specificity of ELISA from 65 to $83 \%$ [36]. The combination of functional and antigen assays has the highest performance in terms of specificity and sensitivity [3]. Laboratory assays may help to establish the diagnosis of HIT. However, not all PF4/ heparin antibodies are pathologic. Only a subset of patients with positive antigen assays has platelet activating antibodies, of which only a few patients develop thrombocytopenia and subsequent thrombosis [37]. Hence, this spectrum can be described as an iceberg model', with clinical HIT as the tip of the iceberg [37]. The diagnosis of HIT should be established taking into consideration clinical manifestations and laboratory evidence [38].

\section{Management of patients with HIT General measures}

If there is a clinical suspicion of HIT, all heparin should be stopped, including heparin used to 'flush' intravascular catheters, and regional use for dialysis and to coat catheters $[16,19]$. In patients with strongly suspected or confirmed HIT who do not have active bleeding, prophylactic platelet transfusions are not indicated because this will lead to subsequent platelet activation and increased risk of thrombosis without a net increase in platelet count [16].

\section{Alternative anticoagulation}

The highest risk of new, progressive, or recurrent thrombosis occurs in the first few days after stopping heparin. Alternative therapeutic anticoagulation should be initiated based on a high clinical suspicion and not delayed while awaiting confirmatory laboratory testing or because of thrombocytopenia [39]. When treatment was delayed pending laboratory confirmation of the diagnosis within a clinical trial setting, the incidence of new thrombosis was approximately tenfold higher than during the subsequent period of treatment with a direct thrombin inhibitor [40]. Therapeutic options include direct thrombin inhibitors and factor Xa inhibitors. Oral thrombin and factor Xa inhibitors, such as dabigatran, rivaroxaban und apixaban, are not yet approved in patients with HIT [18]. The choice of alternative anticoagulant depends upon availability, associated medical conditions, and the preference of the medical staff.

\section{Heparinoids}

These are direct factor Xa inhibitors, of which danaproid is the only available preparation established for use in patients with HIT. Danaproid is a mixture of low molecular sulphated gylcosaminoglycans: Heparan, dermatan, and chondroitin sulfate [18]. It inhibits thrombin formation primarily through inhibition of factor Xa. The anti-Xa activity of danaparoid has a halflife of 24 hours. The bioavailability reaches almost 100\% after intravenous or subcutaneous administration with a reliable dose-response curve. Anti-Xa levels (target, 0.50.8 anti-Xa $\mathrm{U} / \mathrm{ml}$ ) may be used to guide danaparoid therapy. Monitoring anti-factor Xa activity is important in patients with renal dysfunction, as danaparoid is partially excreted in the urine [3]. To avoid overdosage, monitoring of factor Xa activity is also recommended in patients with extremely low or high body weight, lifethreatening thrombosis, bleeding complications, and in critically ill patients with marked organ dysfunction or comorbidity [3].

Cross reactivity with HIT IgG antibody may occur in less than $10 \%$ of cases and cannot be predicted by in vitro testing prior to onset of therapy [41]. Danaparoid blocks HIT antibody-induced platelet aggregation and thromboxane B2 production and has been shown to be an effective alternative anticoagulant in patients with HIT [41]. In some instances, HIT IgG antibody's cross reaction may have clinical consequences. Laboratory testing for cross reactivity should be reserved, however, 
for suspected cases, such as those who develop thrombotic complications during danaparoid therapy or when thrombocytopenia persists for more than 4 days after onset of therapy. Another anticoagulant should be considered if the diagnosis is confirmed [3,41]. The disadvantage of danaparoid therapy is its relatively prolonged kinetics in the absence of a specific antidote. Overdosage, as manifested by increased anti factor Xa activity (> $2 \mathrm{IE} / \mathrm{ml}$ ) may lead to serious bleeding complications and increased mortality rates [4]. Adequate dosing and monitoring of patients at risk is, therefore, mandatory to avoid subsequent complications. Danaparoid was withdrawn from the US market in April 2002, but remains available for treatment and/or prevention of HIT-thrombosis in several other jurisdictions, e.g., Canada, Europe, Japan, Australia, and New Zealand.

\section{Direct thrombin inhibitors}

These substances directly inhibit thrombin. Lepirudin, argatroban und bivalirudin are available for use in patients with HIT.

- Lepirudin: Lepirudin is a recombinant hirudin (found in the saliva of the medicinal leech, Hirudo medicinalis) derived from genetically produced yeasts. It is approved and available in the USA, Canada, Europe, and Australia for treatment of thrombosis complicating HIT [42]. Lepirudin binds to thrombin and inhibits its prothrombotic activity. The activated partial thromboplastin time (aPTT) should be targeted at 1.5 to 2.0 times the patient's baseline aPTT or the mean laboratory normal range. After intravenous administration, lepirudin reaches a peak level within 15 minutes and plasma levels maintain a steady state for $1-2$ hours $[3,43]$. To avoid overdosage and bleeding complications some authors suggest starting intravenous infusion without bolus administration, unless fulminant thrombosis is present [3]. Dose adjustment is required in patients with renal dysfunction, as the drug undergoes renal elimination.

In $30 \%$ of patients who are treated with lepirudin, antihirudin IgG antibodies may develop. This was not found to be associated with higher risk of thrombosis, bleeding, or anaphylactic reactions, thus, lepirudin administration should not be discontinued for this reason [3]. Anaphylactic reactions due to lepirudin therapy are rarely observed. The risk of anaphylaxis can be reduced by avoiding bolus doses [3]. The risk of bleeding was also found to be increased with simultaneous use of acetylsalicylic acid [44]. Therefore, acetylsalicylic acid therapy should be avoided during concomitant therapy with direct thrombin inhibitors.

- Argatroban: Argatroban is a synthetic L-arginine derivative. It reversibly inhibits both soluble and clotbound thrombin and has a half-life of 50 minutes after intravenous administration. The infusion rate should be adjusted to target the aPTT at 1.5 to 3 times of initial levels. Reduced infusion rates are appropriate in patients with heart failure, multiple organ system failure, severe anasarca, and during the early postcardiac surgery period. In patients with hepatic dysfunction, the half-life increases up to 6 hours, as argatroban undergoes hepatobiliary excretion [3]. This is particularly relevant in ICU patients because of the common occurrence of hepatic perfusion abnormalities in the ICU setting $[3,45]$. Argatroban is contraindicated in patients with liver cell failure $[3,45]$. The advantage of argatroban over LMWH and danparoid is the absence of cross reactivity, as argatroban does not posses molecular similarity to heparin. In addition, antibody formation does not occur, which is an advantage compared to lepirudin [46]. Nevertheless, the incidence of thromboembolic complications was shown to be higher in patients treated with argatroban than in those who were treated with lepirudin or danaparoid. This was explained, however, by the shorter duration of argatroban therapy compared to lepirudin [40].

- Bivalirudin: Bivalirudin is a synthetic congener of hirudin. It exerts its anticoagulant effect through direct thrombin inhibition. The half-life of bivalirudin is 25 minutes after intravenous administration and increases to up to 4 hours in patients with renal failure undergoing dialysis. Only $20 \%$ of the drug is excreted in the urine, whereas $80 \%$ undergoes enzymatic proteolysis [47]. Bivalirudin therapy can be monitored by aPTT or the activated clotting time (ACT). In the absence of a specific antidote, hemofiltration, hemodialysis, or plasmapharesis may be effective therapeutic options [48]. This drug is approved in the USA, Canada, Europe, Australia, New Zealand, and Latin America for anticoagulation during percutaneous transluminal coronary intervention (PCI); in the USA it is also approved for PCI with provisional use of glycoprotein IIb/IIIa antagonist therapy, and for patients with, or at risk of HIT (or HIT with thrombotic complications) undergoing PCI; it is also approved in Canada for patients with, or at risk of HIT (or HIT with thrombosis syndrome) undergoing cardiac surgery. In Germany, bivalirudin is not approved in HIT patients but is used 'off-label' in special situations, such as anticoagulation during cardiac surgery in patients with HIT [47].

The anticoagulant effect of bivalirudin is similar to other available alternative anticoagulants with a reduced risk of bleeding [47]. In patients with renal or hepatic dysfunction, bivalirudin therapy is advantageous, as it undergoes enzymatic proteolysis in addition to renal excretion. Cross reactivity with HIT antibodies has not been reported [3]. 


\section{Fondaparinux}

Fondaparinux is a synthetic, heparin analog, pentasaccharide anticoagulant. It enhances factor Xa inhibition by binding to antithrombin III. The half-life is 18 hours, so that it should be administered only once per day [49]. The dose response curve is linear. Fondaparinux undergoes predominant renal excretion and is, therefore, contraindicated in patients with terminal renal failure [50]. The anticoagulant effect of fondaparinux is more potent than enoxaparin [49]. The risk of bleeding is not increased as compared to LMWH [49].

Patients treated with fondaparinux develop anti-PF4/ heparin antibodies with a similar frequency to those treated with LMWH [12], but fondaparinux-induced HIT appears to be exceptionally rare. This is probably because of its short polysaccharide chain of 10-12 saccharides and subsequently weak platelet activating potential [50]. In the USA, fondaparinux is approved in patients with HIT for prophylaxis and treatment of thromboembolic diseases. In Germany, this drug is approved for prophylaxis after major orthopedic surgery. The anti-factor Xa activity of fondaparinux can be useful in patients under warfarin therapy who require alternative anticoagulation preoperatively. The risk of microvascular thrombosis and lower limb gangrene is increased in some patients with HIT who receive concomitant therapy with warfarin and direct thrombin inhibitors (vide infra) [50]. Fondaparinux can be used, therefore, in the transient phase until warfarin therapy is withdrawn, to reduce the risk of thromboembolic complications in these patients [50]. The use of fondaparinux in ICU patients with renal insufficiency and multiorgan failure is not recommended because of the significant risk of accumulation [3].

\section{Heparin and vitamin K antagonist therapy in patients with HIT}

To avoid possible bleeding complications with alternative anticoagulants in the absence of specific antidotes, patients with known HIT may be treated with heparin for short periods, such as those undergoing surgery using a heart-lung machine [6]. However, this should only be considered when heparin has not been administered within the previous 100 days to avoid the occurrence of rapid onset HIT. In addition, the presence of anti-HIT antibodies should be excluded and use of heparin should be avoided during the perioperative period [6].

Vitamin $\mathrm{K}$ antagonist therapy is contraindicated in patients with HIT because of the increased risk of thrombosis in the presence of thrombocytopenia. In these patients, vitamin $\mathrm{K}$ antagonist therapy may induce lower limb venous gangrene or severe skin necrosis. This occurs a few days after the onset of vitamin $\mathrm{K}$ antagonist therapy $[39,51]$. Treatment with vitamin $\mathrm{K}$ antagonists is associated with a rapid decrease in protein $\mathrm{C}$ concentration, which has a short half-life of 6 hours, whereas serum levels of procoagulant coagulation factors (Factors II, VII, $\mathrm{IX}, \mathrm{X}$ ) remain high during the first days of therapy. This imbalance between pro- and anticoagulant favors a prothrombotic state [4]. Use of vitamin $\mathrm{K}$ antagonist therapy should be postponed until the platelet count has recovered substantially and, thereafter, started at a low dose [16]. Alternative anticoagulants should be used during thrombocytopenia and should be continued until the platelet count has reached a stable plateau and the international normalized ratio (INR) has reached the intended target range, with a minimal overlap of 5 days $[14,16]$. For patients receiving a vitamin $K$ antagonist at the time of diagnosis of HIT, use of vitamin $\mathrm{K}$ is recommended $[16,39,51]$.

\section{The challenge of diagnosis and treatment of HIT in the ICU}

Anti-thrombosis prophylaxis is a keystone in the management of critically ill patients. In German ICUs, $99 \%$ of patients receive prophylactic anticoagulants, $88 \%$ receive $\mathrm{LMWH}$ and $45 \%$ receive UFH during the ICU stay [52]. HIT is, therefore, a major concern in ICU patients. Nevertheless, thrombocytopenia is a common occurrence in $30-50 \%$ of ICU patients, so that diagnosis of HIT represents a major challenge [53]. Common reasons for thrombocytopenia in these patients include sepsis, adverse effects of drugs, transfusion reactions, and major surgical procedures [54]. The development of thrombotic complications under heparin therapy is, therefore, a better indicator of a diagnosis of HIT than uncomplicated thrombocytopenia [3]. Repeated occlusion of hemodialysis filters and necrotic or erythematous skin lesions at the site of heparin injections may also be an important sign of HIT $[3,53]$. In this context, the use of HIT scores, such as the 4 T's score, may be helpful in establishing the diagnosis (vide supra). In uncomplicated cases with a low probability of HIT, heparin administration should not be discontinued and further laboratory testing is not required. In patients where HIT is moderately or strongly suspected, heparin administration should be stopped pending the results of laboratory testing, and alternative anticoagulation should be initiated [3]. Even though platelet activation (functional) assays are more specific for detecting HIT antibodies than antigen tests, neither test is completely specific for HIT, which is considered a clinicopathological syndrome (vide supra).

\section{Summary and conclusion}

HIT is an immune-mediated, prothrombotic complication that occurs with UFH and to a lesser extent with LMWH. HIT is a clinicopathologic syndrome with one or more clinical events (thrombocytopenia with or without 
thrombosis). The diagnosis of HIT can be established by laboratory testing for the presence of HIT antibodies. The combination of functional and antigen assays has the highest performance in terms of specificity and sensitivity. Alternative therapeutic anticoagulation should be initiated based on high clinical suspicion and not delayed while waiting for confirmatory laboratory testing or because of thrombocytopenia. Therapeutic options include direct thrombin inhibitors and factor Xa inhibitors. The choice of alternative anticoagulant depends upon availability, associated medical conditions, and preferences of the medical staff. The diagnosis of HIT in the ICU is a major challenge as thrombocytopenia is prevalent in these patients and generally caused by conditions other than HIT. In this context, the use of the 4 T's score may be helpful in establishing the diagnosis and management of these patients.

\section{Competing interests}

The authors declare that they have no competing interests.

\section{List of abbreviations used}

aPTT: activated partial thromboplastin time; CAMP: cyclic adenosine monophosphate; HIPA: heparin-induced platelet activation; HIT: heparininduced thrombocytopenia; LMWH: low-molecular-weight-heparin; PaGIA: particle gel immunoassay; PIFA: particle immunofiltration assay; PF4: platelet factor 4; SRA: serotonin release assays; UFH: unfractionated heparin.

Published: 22 March 2011

\section{References}

1. Thong CL, Kam PCA: Heparin-induced thrombocytopenia. Curr Anaesth Crit Care 2005, 16 143-150

2. Greinacher A, Eichler P, Lietz T, Warkentin TE: Replacement of unfractionated heparin by low-molecular-weight heparin for postorthopedic surgery antithrombotic prophylaxis lowers the overall risk of symptomatic thrombosis because of a lower frequency of heparin-induced thrombocytopenia. Blood 2005, 106:2921-2922.

3. Selleng K, Warkentin TE, Greinacher A: Heparin-induced thrombocytopenia in intensive care patients. Crit Care Med 2007, 35:1165-1176.

4. Picker SM, Gathof BS: Heparin induced thrombocytopenia. A frequently unrecognised complication after major orthopedic surgery. Orthopade 2004, 33:1300-1308

5. Battistelli S, Genovese A, Gori T: Heparin-induced thrombocytopenia in surgical patients. Am J Surg 2010, 199:43-51.

6. Greinacher A, Warkentin TE: Recognition, treatment, and prevention of heparin-induced thrombocytopenia: review and update. Thromb Res 2006, 118:165-176.

7. Amiral J, Peynaud-Debayle E, Wolf M, Bridey F, Vissac AM, Meyer D: Generation of antibodies to heparin-PF4 complexes without thrombocytopenia in patients treated with unfractionated or lowmolecular-weight heparin. Am J Hematol 1996, 52:90-95.

8. Aster RH: Heparin-induced thrombocytopenia and thrombosis. N Engl J Med 1995, 332:1374-1376.

9. Shoenfeld Y, Praprotnik S, Levy Y, et al:: Pathogenic anti-endothelial cell antibodies: classification to anti-microvascular EC and anti-macrovascular EC antibodies. Isr Med Assoc J 2000, (2 Suppl):24-25.

10. Khairy M, Lasne D, Amelot A, et al:: Polymorphonuclear leukocyte and monocyte activation induced by plasma from patients with heparininduced thrombocytopenia in whole blood. Thromb Haemost 2004, 92:1411-1419.

11. Davidson SJ, Wadham P, Rogers L, Burman JF: Endothelial cell damage in heparin-induced thrombocytopenia. Blood Coagul Fibrinolysis 2007, 18:317-320.

12. Warkentin TE, Cook RJ, Marder VJ, et al:: Anti-platelet factor 4/heparin antibodies in orthopedic surgery patients receiving antithrombotic prophylaxis with fondaparinux or enoxaparin. Blood 2005, 106:3791-3796.

13. Arepally $G M$, Hursting MJ: Platelet factor $4 /$ heparin antibody $(\mathrm{lg} G / \mathrm{M} / \mathrm{A})$ in healthy subjects: a literature analysis of commercial immunoassay results. J Thromb Thrombolysis 2008, 26:55-61.

14. Hassan Y, Awaisu A, Aziz NA, Aziz NH, Ismail O: Heparin-induced thrombocytopenia and recent advances in its therapy. J Clin Pharm Ther 2007, 32:535-544.

15. Lubenow N, Kempf R, Eichner A, Eichler P, Carlsson LE, Greinacher A: Heparininduced thrombocytopenia: temporal pattern of thrombocytopenia in relation to initial use or reexposure to heparin. Chest 2002, 122:37-42.

16. Warkentin TE, Greinacher A, Koster A, Lincoff AM: Treatment and prevention of heparin-induced thrombocytopenia: American College of Chest Physicians Evidence-Based Clinical Practice Guidelines (8th Edition). Chest 2008, 133:340S-380S

17. Warkentin TE, Sheppard JA, Moore JC, Moore KM, Sigouin CS, Kelton JG: Laboratory testing for the antibodies that cause heparin-induced thrombocytopenia: how much class do we need? J Lab Clin Med 2005, 146:341-346.

18. Warkentin TE: Heparin-induced thrombocytopenia. Hematol Oncol Clin North Am 2007, 21:589-607.

19. Shantsila E, Lip GY, Chong BH: Heparin-induced thrombocytopenia. A contemporary clinical approach to diagnosis and management. Chest 2009, 135:1651-1664.

20. Warkentin TE, Chong BH, Greinacher A: Heparin-induced thrombocytopenia: towards consensus. Thromb Haemost 1998, 79:1-7.

21. Warkentin TE, Crowther MA: When is HIT really HIT? Ann Thorac Surg 2007 83:21-23.

22. Greinacher A, Eichler P, Lubenow N, Kwasny H, Luz M: Heparin-induced thrombocytopenia with thromboembolic complications: meta-analysis of 2 prospective trials to assess the value of parenteral treatment with lepirudin and its therapeutic aPTT range. Blood 2000, 96:846-851.

23. Cawley MJ, Wittbrodt ET, Boyce EG, Skaar DJ: Potential risk factors associated with thrombocytopenia in a surgical intensive care unit. Pharmacotherapy 1999, 19:108-113.

24. Vanderschueren S, De Weerdt A, Malbrain M, et al:: Thrombocytopenia and prognosis in intensive care. Crit Care Med 2000, 28:1871-1876.

25. Drews RE: Critical issues in hematology: anemia, thrombocytopenia, coagulopathy, and blood product transfusions in critically ill patients. Clin Chest Med 2003, 24:607-622.

26. Greinacher A, Farner B, Kroll H, Kohlmann T, Warkentin TE, Eichler P: Clinical fea tures of heparin-induced thrombocytopenia including risk factors for thrombosis. A retrospective analysis of 408 patients. Thromb Haemost 2005, 94:132-135.

27. Linkins LA, Warkentin TE: The approach to heparin-induced thrombocytopenia. Semin Respir Crit Care Med 2008, 29:66-74.

28. Pohl C, Klockgether T, Greinacher A, Hanfland P, Harbrecht U: Neurological complications in heparin-induced thrombocytopenia. Lancet 1999, 353:1678-1679.

29. Wester JP, Haas FJ, Biesma DH, Leusink JA, Veth G: Thrombosis and hemorrhage in heparin-induced thrombocytopenia in seriously ill patients. Intensive Care Med 2004, 30:1927-1934.

30. Warkentin TE: Heparin-induced thrombocytopenia. Dis Mon 2005, 51:141-149.

31. Warkentin TE, Heddle NM: Laboratory diagnosis of immune heparin induced thrombocytopenia. Curr Hematol Rep 2003, 2:148-157.

32. Lo GK, Juhl D, Warkentin TE, Sigouin CS, Eichler P, Greinacher A: Evaluation of pretest clinical score (4T's) for the diagnosis of heparin-induced thrombocytopenia in two clinical settings. J Thromb Haemost 2006, 4:759-765.

33. Schallmoser K, Drexler $C$, Rohde E, et al:: The particle gel immunoassay as a rapid test to rule out heparin-induced thrombocytopenia? J Thorac Cardiovasc Surg 2009, 137:781-783.

34. Greinacher A, Gopinadhan M, Gunther JU, et al:: Close approximation of two platelet factor 4 tetramers by charge neutralization forms the antigens recognized by HIT antibodies. Arterioscler Thromb Vasc Biol 2006, 2386-2393.

35. Shaheed G, Malkovska V, Mendoza J, et al.: PF4 ENHANCED assay for the diagnosis of heparin-induced thrombocytopenia in complex medical and surgical patients. Crit Care Med 2007, 35:1691-1695.

36. Parker Rl: Measurement of heparin-dependent platelet antibodies in the diagnosis of heparin-induced thrombocytopenia: fact or fiction? Crit Care Med 2007, 35:1784-1785. 
37. Warkentin TE: Heparin-induced thrombocytopenia: pathogenesis and management. Br J Haematol 2003, 121:535-555.

38. Warkentin TE: Heparin-induced thrombocytopenia: a clinicopathologic syndrome. Thromb Haemost 1999, 82:439-447.

39. Warkentin TE, Greinacher A: Heparin-induced thrombocytopenia: recognition, treatment, and prevention: the Seventh ACCP Conference on Antithrombotic and Thrombolytic Therapy. Chest 2004, 126:311S-337S.

40. Lubenow N, Eichler P, Lietz T, Greinacher A: Lepirudin in patients with heparin-induced thrombocytopenia - results of the third prospective study (HAT-3) and a combined analysis of HAT-1, HAT-2, and HAT-3. JThromb Haemost 2005, 3:2428-2436.

41. Tardy-Poncet B, Wolf M, Lasne D, et al.: Danaparoid cross-reactivity with heparin-induced thrombocytopenia antibodies: report of 12 cases. Intensive Care Med 2009, 35:1449-1453.

42. Warkentin TE: Management of heparin-induced thrombocytopenia: a critical comparison of lepirudin and argatroban. Thromb Res 2003, 110:73-82.

43. Greinacher A: Lepirudin: a bivalent direct thrombin inhibitor for anticoagulation therapy. Expert Rev Cardiovasc Ther 2004, 2:339-357.

44. Tardy-Poncet B, Tardy B, Reynaud J, et al.: Efficacy and safety of danaparoid sodium (ORG 10172) in critically ill patients with heparin-associated thrombocytopenia. Chest 1999, 115:1616-1620.

45. Keegan SP, Gallagher EM, Ernst NE, Young EJ, Mueller EW: Effects of critical illness and organ failure on therapeutic argatroban dosage requirements in patients with suspected or confirmed heparin-induced thrombocytopenia. Ann Pharmacother 2009, 43:19-27.

46. Lewis $B E$, Wallis $D E$, Berkowitz $S D$, et al:: Argatroban anticoagulant therapy in patients with heparin-induced thrombocytopenia. Circulation 2001, 103:1838-1843.
47. Warkentin TE, Greinacher A, Koster A: Bivalirudin. Thromb Haemost 2008, 99:830-839

48. Koster A, Dyke CM, Aldea G, et al.: Bivalirudin during cardiopulmonary bypass in patients with previous or acute heparin-induced thrombocytopenia and heparin antibodies: results of the CHOOSE-ON trial. Ann Thorac Surg 2007, 83:572-577.

49. Lobo B, Finch C, Howard A, Minhas S: Fondaparinux for the treatment of patients with acute heparin-induced thrombocytopenia. Thromb Haemost 2008, 99:208-214.

50. Warkentin TE: Fondaparinux versus direct thrombin inhibitor therapy for the management of heparin-induced thrombocytopenia (HIT)-bridging the River Coumarin. Thromb Haemost 2008, 99:2-3.

51. Warkentin TE: Should vitamin K be administered when HIT is diagnosed after administration of coumarin? J Thromb Haemost 2006, 4:894-896.

52. Hilbert P, Teumer P, Stuttmann R: Prevention of thromboembolism in German intensive care units: Results of a nationwide survey. Anaesthesist 2008, 57:242-250,

53. Crowther MA, Cook DJ, Meade MO, et al.: Thrombocytopenia in medicalsurgical critically ill patients: prevalence, incidence, and risk factors. J Crit Care 2005, 20:348-353.

54. Baldwin ZK, Spitzer AL, Ng VL, Harken AH: Contemporary standards for the diagnosis and treatment of heparin-induced thrombocytopenia (HIT). Surgery 2008, 143:305-312.

doi:10.1186/cc9993

Cite this article as: Sakr Y: Heparin-induced thrombocytopenia in the ICU: an overview. Critical Care 2011, 15:211. 